Open Access to Pharmaceutical and Medical Research

(C) 2011-18, publisher and licensee JDDT, This is an Open Access article which permits unrestricted non-commercial use, provided the original work is properly cited

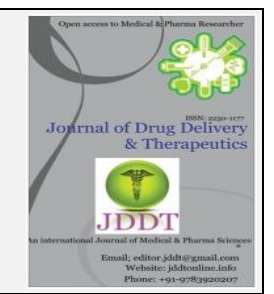

Open $\mathcal{O}_{\text {Access }}$

Review Article

\title{
Inflammatory Bowel Disease and Diabetes: An Association in Horizon
}

\author{
Avik Das ${ }^{1 *}$, Srikanta Chandra ${ }^{2}$, Jyotirmay Samanta ${ }^{3}$, Preeta Bose ${ }^{4}$, Tathagata Roy ${ }^{5}$, Lucky Mukherjee 6 \\ 1. Assistant Professor of Gupta College of Technological Sciences , Department of Pharmacology, Asansol, West Bengal, 713301 \\ 2. Assistant Professor of Jakir Hossain Institute of Pharmacy, Raghunathganj, Murshidabad, 742225 \\ 3. Assistant Professor of Genex College of Pharmaceutical Science \& Technology, Department of Pharmacology, Hooghly, Chinsurah, 712102 \\ 4. Department of Pharmaceutical Technology, JIS University, Kolkata, 700109 \\ 5. Department of Pharmaceutical Technology , JIS University, Kolkata , 700109 \\ 6. Assistant Professor of PGMS, Department of Pharmaceutics, Medinipur, 721253
}

\section{ABSTRACT}

As we know inflammatory bowel disease is an emergent plight in rural and developing countries. In USA 10 million of peoples are suffering from IBD.IBD encompasses two pivotal kinds of pathological condition Ulcerative colitis (UC) and Crohn's disease (CD). Epidemiologically has a greater prevalence in the global prospective. So we decided to zero in on IBD in our work ahead. So a thorough search of available literature IBD is associated with plethora of comorbid disorders which includes gastrointestinal disorders arising from cholelithiasis, cutaneous disease like psoriasis, metabolic disorders like diabetes mellitus.

Keywords: IBD: inflammatory bowel disease, CD: Crohn's disease, UC: Ulcerative colitis,

Article Info: Received 14 June 2019; $\quad$ Review Completed 11 August 2019; $\quad$ Accepted 18 August $2019 ; \quad$ Available online 25 August 2019

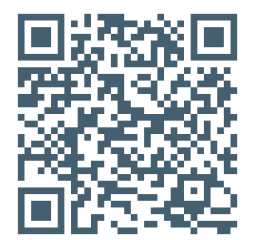

Cite this article as:

Das A, Chandra S, Samanta J, Bose P, Roy T, Mukherjee L, Inflammatory Bowel Disease and Diabetes: An Association in Horizon, Journal of Drug Delivery and Therapeutics. 2019; 9(4-s):796-799 http://dx.doi.org/10.22270/jddt.v9i4s.3423

Avik Das, Assistant Professor of Gupta College of Technological Sciences, Department of Pharmacology, Asansol, West Bengal, 713301

\section{Introduction:}

As we know Inflammatory Bowel Disease (IBD) is an emergent plight in developed \& developing countries. According to American Gastrological Society (AGS) Inflammatory Bowel Disease (IBD) is defined as acute or chronic idiopathic ulceroinflammatory condition of the bowel which may or may not have transmural stretch Inflammatory Bowel Disease (IBD) encompasses two pivotal kinds of pathological condition one is Ulcerative Colitis (UC) and Crohn's Disease(CD) . Ulcerative Colitis (UC) is a chronic ulceroinflammatory condition primarily confined within colonic mucosa with variable distortion of the colonic architecture .Whereas Crohn's disease is an ulceroinflammatory condition analogues to ulcerative colitis but having a transmural infestation. Since the featured encountered in the clinical course of the disease are multiferous spanning from rectal bleeding, weight loss, abdominal pain and malaise.[1] In the year of 1875 two scientists are discovered namely Wilks \& Moxon, who distinguished it from diarrheal diseases caused by infectious agents. However, in the year of 1932, three doctors narrowing down on Crohn's disease (CD) namely Burnil 1
Crohn, Leon Ginzberg and Gordon . D oppenheimer. Since many theories have been explored behind rationale of Ulcerative colitis (UC) .People who are suffering from ulcerative colitis (UC) shows abnormality of the immune system. Pivotal point is that Ulcerative colitis is not caused by emotional distress or sensitivity to certain foods. It is prevalent in any age but start mostly between ages $15-30$ or $50-70$. Inflammatory bowel disease (IBD) is associated with a plethora of comorbid disorder such as gastrointestinal disorder arising from cholelithiasis, cutaneous disease like psoriasis \& metabolic disorders like diabetes mellitus .Coming to the option available for management they include both non pharmacological approach \& pharmacological approach . Under non pharmacological approach life style modification such as monitored dieting, exercise may plays a desire role in the therapy and psychological intervention also deployed to control the functional aspect of the disease. On the other hand Salfasalazine and Balsalazide are still considered as a corner stone of the therapy as per as pharmacological approach . [2] 


\section{Epidemiology:}

Inflammatory bowel disease (IBD) is looming large on the modern society which has raised the brows of the medical scientists in both the developed and developing countries. There is wide variation between the incidence rates of Inflammatory Bowel Disease (IBD) In Europe country incidence rates range from $4.1 / 100,000$ (Romania) to 81.5/100.000. In Asia and the Middle East incidence was lower $0.1-6.3 / 100.000$. On the other hand in USA the incidence is having intermediates rates ranging from 0 19.2/100.000 population. [3] However currently in Sweden 61,000 patients are suffering from Inflammatory Bowel Disease (IBD) . In Brazil an epidemiological study conducted by the Botucatu Medical School Evaluated the incidence and prevalence of Inflammatory bowel disease in micro region of Sao Paulo State. Since, in India the first such study was conducted by Khosla et al., in the year of 1984 at Haryana in North India.[4] The study included 21,971 participants and noted a prevalence of 42.8 Ulcerative colitis (UC) patients. [5] The next study which is conducted in 15 years later by Sood et al., from Punjab . However, incidence was calculated after 1 year later and was reported to be $6.02 / 100.000$. The crude overall incidence of Inflammatory bowel disease (IBD), Ulcerative colitis (UC) and Crohn's disease(CD) from Asia was $1.37,0.76$ and 0.54 per 100.000 where as in Australia it was 23.67,7.33 and 14.00 respectively bin. Data from western Asia including studies from Kuwait , Turkey and Israel revealed an incidence rate of 2.8/100.000 and 5.04/100.000 in Kuwait and Israel respectively bin. However, the prevalence of 4.9/ 100.000 and 167/100.000 in Turkey and Israel respectively bin. The prevalence of Crohn's disease (CD) in japan appears to have risen very rapidly from 2.9/100.000 individually in the year of 1986 to $13.5 / 100.000$. quadrupled from $7.57 / 100.000$ individually in 1997 to $30.87 / 100.000$ individuals in 2005.Over a period of 9 years the prevalence of Ulcerative colitis in Hong kong nearly tripled from 2.36/100,000 individuals in 1997 to $6.30 / 100,000$ individuals in 2006 . Crohn's disease prevalence in Singapore also increased markedly from $1.3 / 100,000$ individuals in 1990 to 7.2 $/ 100,000$ individuals in2004.[5] The geographical variation is more common for inflammatory bowel disease (IBD) . However, in Sweden and Netherlands pediatric patients who are suffering from Ulcerative colitis that is more common rather than Crohn's disease (CD) .Since, the most instrumental point is that Crohn's disease become more active rather than Ulcerative colitis in Sweden. In Scotland Crohn's disease is not unknown rather than England .[6] In Asian countries specially in India subcontinent the shadow of inflammation bowel disease (IBD) is looming large on the society through the recent past. Crohn's disease may shown below the age of 20-25 years varies between 25-40\% it also affect both sex boys and girls. In South Indian boys are more effected on Crohn's disease rather than South Indian girls. Most studies reporting the incidence of CD (3/5) were from the United Kingdom, with a predominant SA migrant group. The remaining two studies from Canada described the SA pediatric population and one study compared non immigrants to $\mathrm{SA}$. The incidence of $\mathrm{CD}$ in SAs was consistently lower than Caucasian ,except for one Canadian pediatric study .[7]The Benchimol study showed a lower incidence in SA compared to other groups within the same environment .The two United Kingdom studies where the incidence was examined over two time periods showed an increase in the incidence of CD in the SA population ,from 1.2 to $2.3 / 100000$ in East London and 1.2 to $3.1 / 100000$ in Leicester .The last migrant United Kingdom incidence studies were published in 1989.[8]

\section{Pathophysiology of Inflammatory Bowel Disease (IBD): A General Overview}

Epidemiologically ulcerative colitis (UC) has a greater prevalence in the global prospective. so, we decided to zero in on ulcerative colitis in our work ahead. As suggested in the literature the course of ulcerative colitis is often complicated due to the multifactorial etiology under pinning the conditions which demands closer overview The pivotal contours may be featured as toxic mega colon, pseudo polyps , and backwash ileitis. .Toxic mega colon evolve from the stalling of the fecal movement of the colonic lumen due to the spasticity arising from the wide spread neural damage in the region associated with microbial over growth. However, in ulcerative colitis there is wide spread destruction of the luminal wall which leads to formation epithelium bulges obstructing the lumen these are commonly termed as pseudo polyps .On the other hands a common featured essentially associated with pancolitis in which the lesions spread beyond the confinds of ileocecum junction this is known as backwash ileitis. Inflammatory bowel disease (IBD) is a multifactorial disorder characterized by chronic inflammation of the intestine . Since the pivotal point is that disturbance of the immune system or imbalanced interactions with microbes leads to amelioration of chronic intestinal inflammation.Th1 cells play a role in the pathogenesis of Inflammatory bowel disease (IBD) is related to chrocity of intestinal inflammation whereas Th2 cells play a role beyond the pathogenesis of Crohn's disease (CD) . However, after activation of Th17 / regulatory T (Treg cells) are pivotal component which is helped to build up intestinal inflammation. Since, Tumor necrosis factor( TNF) are plays a instrumental role to identify the pathogenesis of Inflammatory bowel disease (IBD) . [8] The intestinal epithelium cells contains different types of cells namely enterocyte, goblet cell, neuroendocrine cell, M cell or mother cell and epithelium resident intestinal stem cells. These cell structurally constitute crypts and villi. A single columnar cell lining with a tight junction and secrete mucus containing antimicrobial peptides, to protect mucosa, a mucus layer is present which is covered by epithelial surface . However, this epithelium mucus layer is composed of glycosaylated mucin, mucin from goblet cells. Since, mucin is encoded by Muc 2 . However, genetic factors plays a role so here mutation of NOD2 gene is a instrumental point in the pathogenesis of inflammatory bowel disease (IBD) . [9] The intestinal epithelium which is a pivotal part of the innate immune system and play a role in the maintenance of mucosal homeostasis. Epithelium cells are very tight and highly selective between periphery and intraluminal microenvironment. Briefly, the chief determinant of the course of pathology include intestinal microbiota, intestinal epithelium dysfunction and aberrant mucosal immune response . However, chromosome 5 containing SLC222A4 and SLC22A5 also shows some aberrations in patients suffering from Crohn's disease (CD) . [10] In the modern era microbial profiles at various stages of colitis have been described and characterized that depend on the time and area within the gastrointestinal tract. It is not yet entirely clear whether changes in the composition of the microbiota are the cause or consequence of inflammatory process in the intestinal tissue. Since, the most consistent change observed among the vast majority of inflammatory bowel disease (IBD) patients is a decrease in intestinal microbiota diversity, with slightly different findings between ulcerative colitis (UC) and Crohn's disease ( CD) patients .Since, in Crohn's disease ( CD) a decrease in Formicates is often observed , including butyrate -producing bacteria namely Faecalibacterium prausnitzii . This leads to over production 
of proinflammatory cytokines and downstream events. In Ulcerative colitis (UC) , several other groups of bacteria besides butyrate producing Firmicutes are often reduced, including Bacteriodes and Clostridium genera. On the other hand, Enterococcus and Gamma proteo bacteria are found in higher amounts in fecal samples from Ulcerative colitis (UC) patients. Although the interaction between the host and intestinal microbiota seen to plays a crucial role in the pathogenesis of inflammatory bowel disease (IBD) .Since , a significant amount of IBD patients do not achieve clinical remission after conventional therapy, there is legitimate need for new therapeutic approaches .[11]

\section{Comorbidities Associated with Inflammatory Bowel Disease: A Brief Overview}

Ulcerative colitis is a plethora of comorbid disorder namely gastrointestinal disorder arising from cholelithiasis, cutaneous disease like psoriasis, metabolic disorder like diabetes mellitus. Psoriasis is a chronic hyper proliferative immune mediated inflammatory skin disease. However, 1$3 \%$ population suffered from this disease. Pathophysiology of psoriasis having different factors are include namely genetic predisposition another pivotal point is that environmental factors which are associated with skin disease . However, different types of immune mediated facts are responsible for pathology of psoriasis. On the other hand $\mathrm{T}$ cell or helper $\mathrm{T}$ cell play a role in the pathology of psoriasis. Cytokine, denditric presenting cell they are play a role in the pathology of psoriasis. Since, the top most factors are that TH1, TH17, TNF alpha , IFN gama , IL 17, IL 22 they are play a major role in the pathology of psoriasois.[12]

Inflammatory bowel disease (IBD) is a group of clinico pathological condition of the gastro intestinal tract. Ulcerative colitis is associated with cardiovascular disorder namely hypertension. Cardiovascular is one of the main reason of death in developing countries and its prevalence is increase with age. The impact of Cardiovascular disorder on Inflammatory and bowel disease is the same as for the general population, increase complication and remaining a common cause of mortality. Venous thrombolism ( VTE) which is a common manifestation now a days and it is directly associated with inflammatory bowel disease( IBD) . Atherosclerosis is a common phenomenon in several immune based inflammatory disease particularly rheumatoid arthritis and systemic vascular system are well known . However, different types of adverse effects are shows like hypertension, hyperglycemia , hyperinsulinemia and hyperlipidemia. However rheumatoid arthritis, inflammatory activity control can reduce cardiovascular complications. Aminosalicylates reduce platelet activation whereas azathioprine inhibits formation of platelet leukocyte aggregate. [13]

Ulcerative colitis is an emergent problem in rural and developing countries. Ulcerative colitis is a chronic ulceroinflammatory condition of the bowel primarily confined with colonic mucosa with variable distortion of the colonic architecture. Ulcerative colitis is associated with cholelithiasis or gall bladder stone or intrahepatic stone or common bile duct stone. However, the reason of association between ulcerative colitis and cholelithiasis are leads to cholecystitis , cholangitis , pancreatitis.[14]

Inflammatory bowel disease (IBD) is a group of inflammatory intestinal disorders comprising two types of disease : Ulcerative colitis another one is Crohn's disease . Ulcerative colitis is a chronic ulceroinflammatory condition primarily confined within colonic mucosa with variable distortion of the colonic architecture. Since , inflammatory bowel disease is associated with peripheral artery disease (PAD) . Traditionally, the incidence of this disease is relatively stable in Western countries ; however the IBD incidence has been increasing in Asian countries in the past few years. Peripheral arterial disease ( PAD) is the narrowing of arteries other than those that supply blood to brain or heart, causing a considerable burden on health care systems worldwide. The prevalence of peripheral vascular disease in the general population is $12 \%$ to $14 \%$, affecting up to $20 \%$ of people $>70$ years . According to the Taiwan National Health Insurance ( NHI) data analysis, the incidence of invasive PAD treatment is increasing. Traditional risk factors for PAD are an older age , male sex, hypertension, diabetes, hyperlipidemia , obesity , smoking and family history of vascular disease. [15]

\section{Association Inflammatory Bowel Disease and Diabetes Mellitus: A Brief Overview}

Inflammatory bowel disease (IBD) is a emergent plight as in the modern era. According to American Gastrological Society Inflammatory bowel disease is an acute or chronic idiopathic ulceroinflammatory condition of the bowel which may or may not have a transmural stretch. Inflammatory bowel disease encompasses two pivotal kinds of pathological condition one is ulcerative colitis( UC) another one is crohn's disease. Ulcerative colitis is a chronic ulceroinflammatory condition of the bowel primarily confined within colonic mucosa with variable distortion of the colonic architecture. However, the featured encountered in the clinical course of the disease are multiferous spanning from rectal bleeding, abdominal pain, weight loss and malaise .Inflammatory bowel disease are associated with diabetes mellitus .They are multifactorial association interventional aggrevession and shared complication. Various factors link diabetes comorbidity to ulcerative colitis .They may range from loss of metabolic homeostasis leading to a disarray in intestinal micro flora and risk of altered responses in the gut brain axis due to several associated complication. Interventional aggrevession is also common as evident from precipitation of hyperglycemia due to chronic use of corticosteroids in prevalent in this patients which may manifest as neurological hepatobiliary , osterioarticular and vascular symptoms.[16]

Cholelithiasis is a very prevalent condition in the general population. Some circumstances explain its increased incidence in inflammatory bowel disease patients, mainly the distortion of bile metabolism induced by the functional and anatomical alterations of the gut. [17]

\section{Conclusion:}

Inflammatory bowel disease is an incurable chronic inflammatory intestinal disorder of the gastrointestinal tract that dramatically impacts quality of life. IBD is associated with a plethora of comorbid disorders. Since, diabetes mellitus \& ulcerative colitis share neurological, hepatobiliary, osteoarticular, vascular and post -operative complications. One of the most common plights is that in diabetic patients with ulcerative colitis is the medical treatment. Corticosteroids which is used for management of ulcerative colitis but its associated with the onset of glucose intolerance and diabetes. 


\section{References:}

1. Atricia Kiesler, Ivan J . Fuss, Warren Strober; Experimental Models of Inflammatory Bowel Diseases; Cellular and Molecular Gastroenterology and Hepatology 2015; 1:154-170

2. Bernard Khor, Agnes Gardet, Ramnik J. Xavier ; Genetics and pathogenesis of inflammatory bowel disease ; International Journal of Science, Vol: 17 , No 2 , 16 June 2011, PP : 307 -317

3. Babickova J , Hyosin A ; Pathological and therapeutic interactions between bacteriophages, microbes and the host in inflammatory disease ; World Journal of Gastroenterology ; Vol :16, No : 2 , 2015

4.Saurabh Kedia, Vineet Ahuj; Epidemiology of Inflammatory Bowel Disease in India: The Great Shift East ; Inflamm Intest Dis2017 ;2:102-115

5. Sonnenberg A, McCarty DJ, Jacobsen SJ, Geographic Variation of Inflammatory Bowel Disease within the United States ; Gastroenterol1991;100:143-149

6. Pinchbeck BR, Kirdeikis J, Thomson ABR; Inflammatory bowel disease in Northern Albert: An Epidemiologic Study. J Clin Gastroenterol 1988;10:505 -515

7. Bram Verstockt, Marc Ferrante, Severine Vermeire, Gert Van Assche ; New treatment option for inflammatory bowel disease ; The Japanese Society of Gastroenterology ,6 $6^{\text {th }}$ February 2018 ,53:585 -590

8. Benjamin Pulli, Muhammad Ali, Reza Forghani, Stefan Schob, Kevin L.C .Hsieh , Gregory Wojtkiewicz, Jenny J. Linnoila , John W. Chen ; Measuring Myeloperoxidase Activity in Biological Samples ; 2013 , July, Volume :8, No : 7 , Page no : 2136-2142

9.Cullen G , Kroshinsky D, Cheifetz AS , Korzenik JR ; Psoriasis associated with anti -tumor necrosis factor therapy in inflammatory bowel disease : a new series and a review of 120 cases from the literature ; Aliment Pharmacol Ther , $2011 \mathrm{Dec}$; 34 (11-12): 1318-27

10. Daniel M. Autenrieth, Daniel C.Baumgart ; Toxic Megacolon, Inflammatory Bowel Disease, Volume 18, No : 3, March 2012

11. Duk Hwan Kim , Jae Hee Cheon ; Pathogenesis of Inflammatory Bowel Disease and Recent Advances in Biologic Therapies ; IMMUNE NETWORK, Vol. 17, No 1: 25-40 February ,2017
12.Dianz Zaidi , E. Wine ; Regulation of Nuclear Kappa -Light 0Enhancer of Activated B Cells in Inflammatory Bowel Diseases ; World Journal of Gastroenterology , 2018 ;V olume :17 , No:2 , Page no : 172-185

13.Efstathios Antoniou, Gerogios Antonios Margonis, Anastasios Angelou, Anastasia Pikouli, Paraskevi Argiri, Ioannis Karavokyros , Apostolos Papalois , Emmanouil Pikoulis; The TNBS -induced colitis animal model : An overview; Annals of Medicine and Surgery 11 (2016) 9-15

14. Giovanni Maconi , Federica Furfaro , RobertabSciurti , Cristina Bezzio , Sandro Ardizzone , Roberto de Frenchis; Glucose intolerance and diabetes mellitus in ulcerative colitis: pathogenetic and therapeutic implications ; World Journal of Gastroenterology 2014 April 7;20(13):3507 -3515

15. Gary R. Lichtenstein, Edward V. Loftus Jr, Kim L. Isaacs , Miguel D. Regueiro, Lauren B. Gerson , Bruce E. Sands; ACG Clinical Guideline : Management of Crohn's Disease in Adults ;American Journal of Gastroenterology 2018; $113: 481$ - 517

16. Giorgos Bamias, Theresa T. Pizarro, Fabio Cominelli; Pathway based approaches to the treatment of inflammatory bowel disease; American Journal of Gastroenterology, 2016 January; 167(1): $104-115$

17. H. Sales -Campos , P.J .Basso , V.B.F. Alves , M.T.C .Fonseca , G Bonfa, V. Nardini , C.R.B. Cardoso; Classical and recent advances in the treatment of inflammatory bowel diseases; Brazilian Journal of Medical and Biological Research (2015) 48(2) : 96 107

18. Hyo Sun Lee, Soo -Kyung Park, Dong I1 Park ; Novel treatments for inflammatory bowel disease ; The Korean Journal of International Medicine, Volume 33 , No :1 January 2018, Page no : $20-27$

19. J.E.KRAWISZ , P.SHARON , W.F . STENSON ; Quantitative Assay for Acute Intestinal Inflammation Based on Myeloperoxidase Activity ;World Journal of Gastroenterology ; Volume 87 , No 6 , 2016, PP :578-598

20. Jaroslaw Nowakowski, Adrian A. Chrobak, Dominika Dudek;Psychiatric illness in inflammatory bowel disease psychiatric comorbidity and biological underpinnings; Psychiatr .Pol .2016;50(6) :1157-1166 